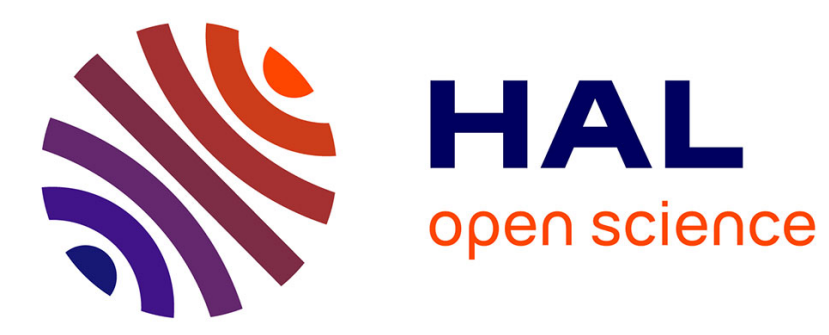

\title{
Millimeter Wave Chipless RFID Authentication based on Spatial Diversity and 2D-Classification Approach
}

Raymundo de Amorim, Romain Siragusa, Nicolas Barbot, Glauco Fontgalland, Etienne Perret

\section{- To cite this version:}

Raymundo de Amorim, Romain Siragusa, Nicolas Barbot, Glauco Fontgalland, Etienne Perret. Millimeter Wave Chipless RFID Authentication based on Spatial Diversity and 2D-Classification Approach. IEEE Transactions on Antennas and Propagation, 2021, 69 (9), pp.5913-5923. 10.1109/TAP.2021.3060126 . hal-03355081

\section{HAL Id: hal-03355081 \\ https://hal.science/hal-03355081}

Submitted on 27 Sep 2021

HAL is a multi-disciplinary open access archive for the deposit and dissemination of scientific research documents, whether they are published or not. The documents may come from teaching and research institutions in France or abroad, or from public or private research centers.
L'archive ouverte pluridisciplinaire HAL, est destinée au dépôt et à la diffusion de documents scientifiques de niveau recherche, publiés ou non, émanant des établissements d'enseignement et de recherche français ou étrangers, des laboratoires publics ou privés. 


\title{
Millimeter Wave Chipless RFID Authentication based on Spatial Diversity and 2D-Classification Approach
}

\author{
Raymundo de Amorim Jr, Student Member, IEEE, Romain Siragusa, Member, IEEE, Nicolas \\ Barbot, Member, IEEE, and Glauco Fontgalland, Senior Member, IEEE and Etienne Perret, Senior Member, IEEE
}

\begin{abstract}
In this paper, a mm-Wave chipless RFID tag is developed to operate in the $V$-band for authentication applications. A novel approach based on tag backscattered E-field measurements at different orientation angles for unitary classification is proposed. The concept is based on the hardness to identically reproduce materials due to the inherent randomness in the fabrication process. These uncertainties are transcribed in very small variations that can be observed in the tag electromagnetic response. A set of 16 identical tags were fabricated, each tag shares same fabrication mask and manufacture process method. Spatial diversity using the tag backscattering pattern (at two different angles) adds independent characteristics for estimating authenticity of each tag. To better exploit the large amount of data collect with this approach, a Machine Learning (ML) sighting classification is used, which enhance the system performance. The probability of error (PE) achieved with the method is around 1\%. This PE is four times lower than the one obtained with a similar approach implemented in the $\mathrm{X}$-band.
\end{abstract}

Index Terms-Authentication technology, Chipless RFID tag, Machine learning (ML), Uniqueness.

\section{INTRODUCTION}

$\mathbf{F}$ ACILITATING and controlling flows, while respecting very strict security constraints is one of the primordial tasks in the supply chain management.Also, this flow management is mainly achieved through the identification and tracking of products and goods used in all industry sectors such as, medicine, food, and textile [1]. Authentication technologies are of great importance to reduce the risk of fraud and counterfeiting. Different characteristics of technologies have been envisaged for this purpose. In this sense, three security levels are usually considered. The first level (overt) relates to characteristics that can be verified easily, such as holograms or color-shift. The second level features are covert, which cannot be easily read without trained examiners using simple instruments, as magnification or UV-lamps. Finally, the third level is described as forensic that provide a high confidence level that can only be viewed in laboratory settings employing

This work was supported by the project AUSTRALE via Agence Nationale de Recherche. (Corresponding author: Raymundo de Amorim Junior.).

The authors are with the Laboratoire de Conception et d'Intégration des Systèmes (LCIS), Grenoble Institute of Technology (Grenoble-INP),Valence 26000, France, (e-mail: raymundo.de-amorim-junior@lcis.grenobleinp.fr; romain.siragusa@lcis.grenoble-inp.fr; nicolas.barbot@lcis.grenobleinp.fr;etienne.perret@lcis.grenoble-inp.fr)

Glauco Fontgalland is with the Universidade Federal de Campina Grande (UFCG), Applied Electromagnetics and Microwave Lab - LEMA, Campina Grande 58428-830, Brazil, (e-mail: fontgalland@dee.ufcg.edu.br). microscopes or other specialized equipment. Then, imaging systems and, in particular, fingerprints were proposed as a solution. However, imaging systems are not secure enough for some authentication applications. For instance, biometric systems only analyze the surface morphology of a fingerprint image, making it possible for hackers to spoof and bypass authentication. Some risks are intrinsic to this application. For instance, the reconstruction of the original biometry from the stored model, the construction of a falsified biometric sample or even a secondary use of biometric information is possible. These factors make this technique fragile despite being quite employed [1]. Smart-chips and identity cards are essentially preferred in the authentication process. The secure storage contains a private key and a card program running in the chip public-key cryptography to generate an encrypted transaction. The cryptography signature has a big data set that cannot be calculated without the knowledge of the private key [2]. This leads to a large time-consuming process for a real-time reading which limits the range of this application.

Contrary to asymmetric cryptography based on computations with an algorithm to encrypt and decrypt code, more recently, researchers have shown the possibility of using electromagnetic waves to retrieve information that is difficult to reproduce and thus make it possible to identify or authenticate objects. The method based on the authentication of individual transmitters/receivers or objects by EM signal characterization are called RF fingerprint identification, which attempts to extract a unique identifier from the received signal. The RF fingerprints identification is performed by the intrinsic characteristics related to hardware and manufacturing randomness, that can be extracted by processing the transient round-trip signal [3]. An approach employing RF fingerprinting is proposed as a solution to identify device model types in [4]. Note also that power amplifier imperfections are used to identify wireless devices in [5], [6]. Authentication based on IEEE 802.15.4 devices using time-domain complex baseband signals were analyzed, the authors demonstrate an identification accuracy of 92.29\% on a set of commercial devices [7]. An RF Certificate of Authentication (RF-CoA) based on substrate variations is proposed in [8], it relies on the near-field RF coupling between a $5 \times 5$ antenna array and the supposed modified substrate of the RF-CoAs. All of these RF fingerprint solutions show that intrinsic and specific information of an object can be carried out using the electric field generated by everyday electronic devices. This relevant information to make authentication can 
have several origins like modulation errors, RF front-end distortion related to pre-processing signal and from various analog devices used in their construction.

The fact that these phenomena are extremely difficult to identically reproduce makes it possible to introduce techniques to reliably know the authenticity of an object. One application example is RFID. Indeed, it has been shown that authentications in passive RFID systems can be implemented using detection of variations in the backscattered signal due to physical variations [9]-[11]. Note that the use of specific protocols has been proposed to improve security level. Traditional passive UHF RFID systems can be adapted to authentication solutions. This represents a lower cost authentication solution given the very significant growth of current RFID technology. However, this functionality of authentication leads to additional blocks in the ASIC implementation, which increases the power consumption, reduces the reading distance, and increases the final cost of the application.

However, due to the considerable economic importance of looking for solutions that are increasingly reliable, low cost, or simply different from those that currently exist, new and original authentication solutions are expected. These shortcomings led to the development of a reliable system, utilizing a noninvasive method based on the exploitation of a distinctiveness signature, which does not need a line of sight and, additionally, it is cost-effective. Chipless RFID technology is promising in that sense. This technology is an intermediate technology between the barcodes and the UHF RFID [12], where chipless tags can be seen as a radar target designed to scatter a specific electromagnetic signature. Two main tags families can be distinguished: the time domain tags [13]-[15] and the frequency domain tags [16]-[19]. Time coding is based on a reflectometry principle; the tag usually consists of a transmission line connected to an antenna. In the frequency coding, the information is directly coded in the variations of the tag backscatter signal spectrum. The ID can be coded based on the scattered signal's amplitude [20], frequency [21], phase [18], or some combination of these parameters. Many applications beyond identification purposes have also been reported [22], [23].

In the field of authentication, chipless RFID technology has been use to authenticate items using a low-cost tag [24][28]. A chipless RFID tag with an authentication purpose was presented in [24], this tag consists of nested concentric annular ring resonators, the randomness characteristics are introduced by the substrate dielectric constant and slot parameters variations due to manufacture process. The Euclidean distance is evaluated for authenticity proof between the signature of design value and the simulated tag signature. The potential of authentication using chipless RFID tags was initially demonstrated in [25] where the randomness in the realizing process of chipless RFID tags was exploited to provide a trustworthy tag's response. In [26], some metrics were analyzed for the characterization of independent parameters in the time domain and frequency domain. A method reported in [27] introduces a PE equivalent to many authentication methods found in the literature. For instance, the reported PE was around 3\%-4\%.

This article presents a method to authenticate RFID chipless tags designed in millimeter wave by exploiting the process variations during tag fabrication taking advantage of the measurement at different angles. In this way, differently from other approaches, this method consists in using aspect-independent parameters contained in the tag backscattering pattern combined with a classification strategy based on Machine Learning (ML) approach.

Contrary to X-band RF approaches [28], millimeter wave range is chosen to increase the tag sensitivity related to uncertainties in the fabrication process. A set of 16 tags based on E-shaped resonators is used to extract a unique signal characteristic to realize the authentication process. Then, the quantitative performance of the method is presented in terms of PE.

The next sections of this paper are described as follow. In Section II, the principle of chipless RFID authentication is detailed. Section III is dedicated to chipless tag design on Vband with consideration on the E-field incidence angle for tag characterization. In section IV the measurement setup and the results are discussed. Section $\mathrm{V}$ provides the authentication process formulation based on the binary class evaluation. Section VI presents the PE of this approach compared with other methods.

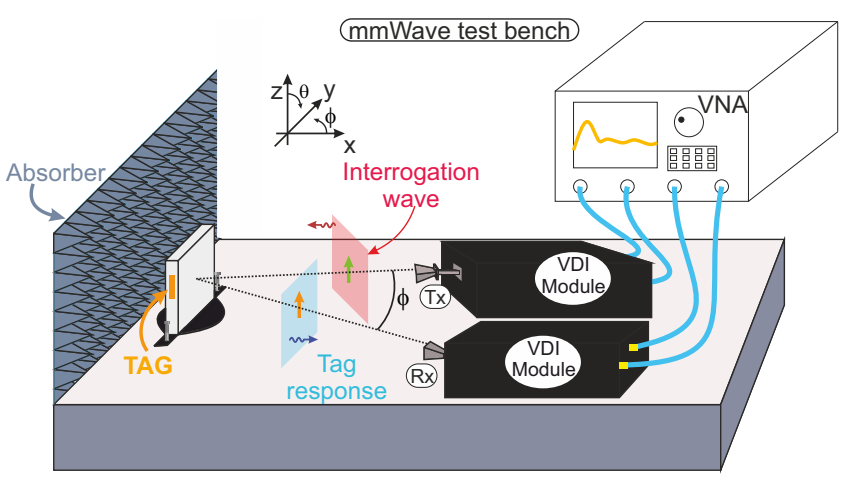

Fig. 1. The mmWave test bench for tag EM measurement.

\section{PRINCIPLE OF CHIPLESS RFID AUTHENTICATION AND TAG DESIGN}

The chipless RFID authentication aims to design a tag that is hardly clonable, i.e. the cost of the counterfeit is much higher than the value of the object attached to it. It means that the tag exhibits a unique, hardly reproducible RF response. Then, this RF footprint must be converted into a digital footprint which contains at least as much information as the total number of objects to authenticate. In this way, the tag's response is measured using the test bench shown in the insets of Fig. 1. The tag's response is measured using a VNA in bi-static configuration. An interrogation wave is sent in far-field configuration to the tag, and then the associated $S_{21}$ parameter associated is measured.

The evaluation of the richness of EM fingerprint information is an essential point for the application, i.e., to quantify the difference that exists between two signals coming from two identical but different realizations of tags. The objective is to quantify this difference which is not noise but something 


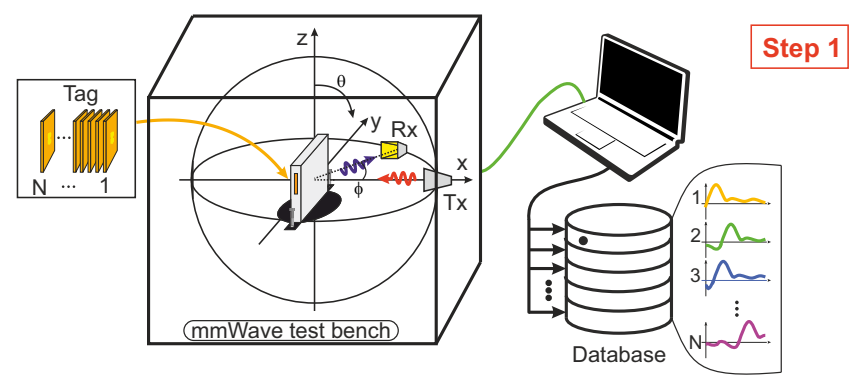

(a)

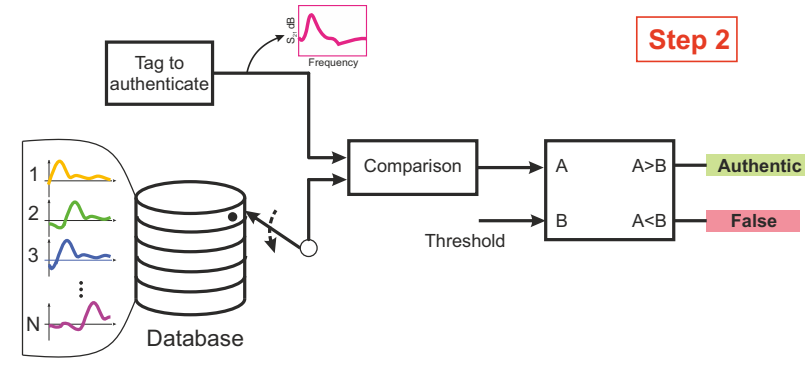

(b)

Fig. 2. Chipless RFID system for authentication. (a) Chipless RFID measurement system on bi-static mode, each chipless tags is characterized, and the corresponding collected RF signatures compose the database, (b) authentication procedure for a chipless RFID system.

related to the tag structure and therefore that can be used to differentiate these two tags. This is relating to the evaluation of aspect-independent parameters inherent to the tag backscattered response. In order to provide a level 3 authentication process, two steps are needed. The first one is carried out after the manufacturing process and the second one when a user needs to authenticate the tag. The formation of the tags' responses database (first step) is detailed in Fig. 2(a). The chipless RFID tags' responses are measured at specific angle for the database enrollment; each $\mathrm{N}$ tag is measured and stored in the database. Compared to [27], each tag is measured at different $\phi$-angles.

The authentication procedure is presented in Fig. 2(b). The EM signal of the unknown tag to authenticate is compared with the previously measured responses of the database. After the comparison, the maximum similarity value resulting from the comparison between the database and the tag to authenticate is held, and then compared with a threshold. If the comparison value (A) is greater than or equal to the threshold (B), then the EM response is authentic or false otherwise.

The authentication approach is based on the idea that the measured signature of the tag presents information related to a random side linked to the way the tag was made. This guarantees the uniqueness of the signature. From the moment one accepts the signature due to randomness, it is not possible to duplicate the tag at a reasonable cost. This is why this authentication method is essentially based on the design of the tag. To show the potential of the approach, the idea is to characterize the probability of authentication error of the introduced tags. This must be done considering the closest possible tags. This is like counterfeiters who would do everything possible to duplicate it and in the best possible conditions. Thus, it is considered that it is possible for the counterfeiters to have access to the dimensions of the tag patterns and that it is possible for them to access to the same fabrication technique as the one we used for the realization of the genuine tags. The approach here goes even further in the sense that we are going to fabricate at the same time a certain number of tags, from the same digital file, next to each other, on the same substrate. The study presented here will consist in characterizing the unique differences that may exist between each tag, differences linked to manufacturing hazards, and to show how to use these RF signals to authenticate these tags. Furthermore, each measured angle adds independent characteristics that will help to determine its authenticity.

In V-band, the size of the resonators is smaller compared to scatters developed in X-band [27]. Consequently, it is expected that the structures will be more sensitive to the physical process error, resulting in better differentiation between the signals backscattered from each tag. This limited size allows the use of multiple resonators to design millimeter wave chipless tags. The strong couplings between resonators will naturally make the structure more sensitive to physical uncertainties. These variations will be related either to inhomogeneous local variations of the materials or to the method of fabrication (errors such as over-etching with chemical etching).

The C-shape scatter is chosen as an elementary particle for the tag. This structure has been widely used in the design and development of chipless RFID tags [19], [20]. The Cshape scatter is shown in Fig. 3. The EM tag resonant response is mainly driven by two main parameters: the spacing between the two arms $(g)$ and the length $(L)$. The over- and under-etching provided by the design tolerance can affect the dimensions of the metal trace of each tag, and hence induce distinct signatures. The resonance frequency of two hypothetical manufacturing structures can be seen in Fig. 3, where $\Delta_{k}$ denotes geometrical variations induced by the manufacturing process. Then, two tags with the same fabrication mask could be affected by different variations $\Delta_{1}$ and $\Delta_{2}$. The resonance frequency is modified by these variations, which makes the whole response sensible to these variations.

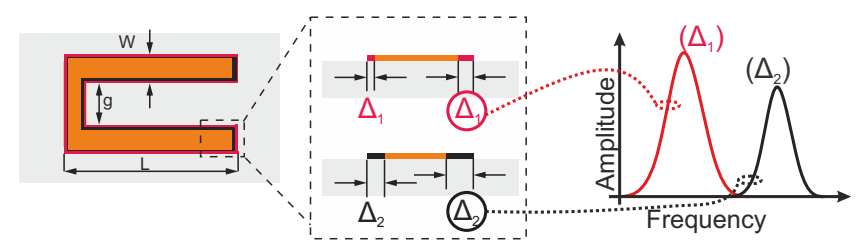

Fig. 3. C-shape scatter with different errors $\Delta_{1}$ and $\Delta_{2}$ imposed by the manufacturing process induce different EM response of the tags. The resonance frequency of the scatter is shifted following these variations. 


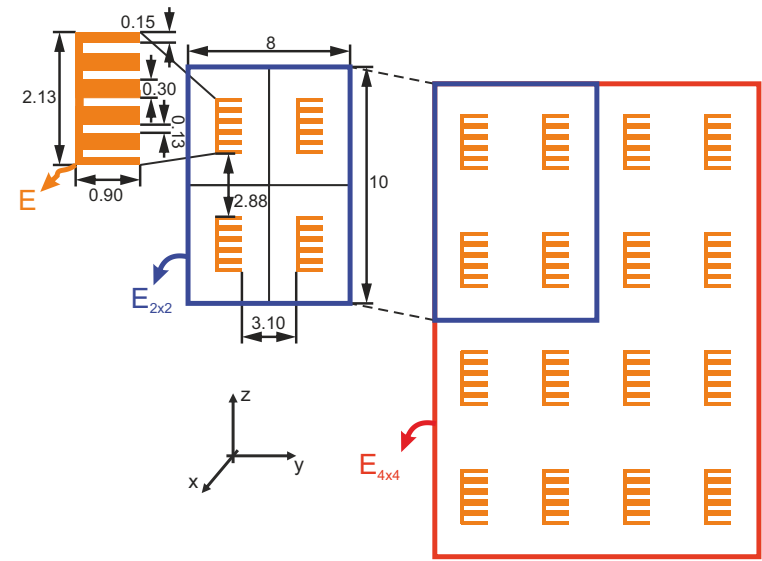

Fig. 4. V-band chipless tag for authentication applications. Multiple resonators are used to increase the RCS scatter level. All the dimensions are in millimeters.

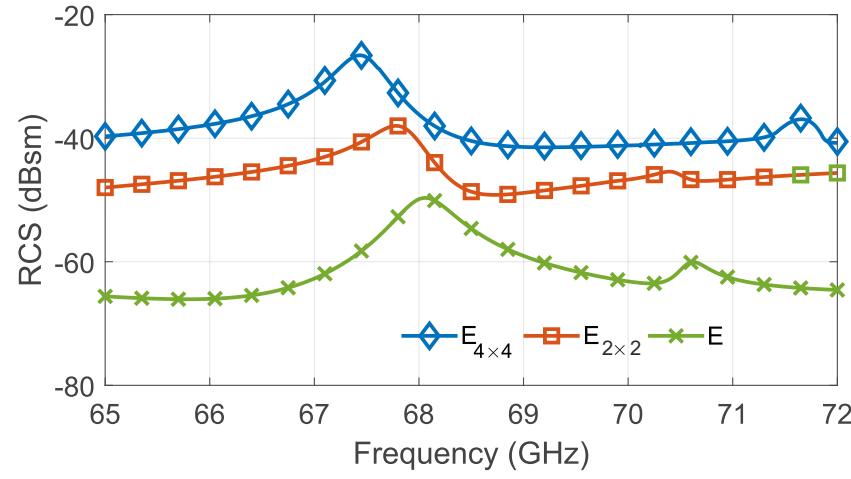

Fig. 5. Simulated RCS versus frequency of different group resonators, where $a \times b$ concerns the lines $a$ and columns $b$ of the network, respectively. For instance, $E_{2 \times 2}$ represents a tag of a group of four $E$ resonators.

In V-band, compared to the X-band, the main limitations are the decrease of the resonator $Q$ factor and the presence of a low RCS level. The RCS of this scatter can be difficult to measure in a real environment. Therefore, to overcome this problem, the same scatter is identically reproduced several times. Thus, to increase the RCS level at a given frequency, the basic principle is to include several times the same resonator on the same tag surface, as depicted in Fig. 4. Furthermore, this increase in the number of resonators will reinforce the coupling between them, which can increase the sensitivity of the structure. Due to coupling, the multiplication of the number of scatters does not carry a proportional increase of the RCS level; hence, an optimization step is performed. The tag's EM responses were obtained by electromagnetic simulation using CST Microwave Studio. The substrate used is the Rogers RT5880 with $\tan \delta=0.0009$, permittivity $\epsilon_{r}=2.33$, and thickness of $0.127 \mathrm{~mm}$. Firstly, one C-shape scatter is analyzed. After, five C-shape scatters are put together without spacing between them forming the E-structure (E in Fig. 4). The quality factor $Q$ of this E-structure is 84.01, compared to 28.03 for the simple C-shape scatters. It is an important metric in this context, since a higher $Q$ represents greater sensitivity due to structural variations. However, whatever its quality factor, to be read correctly, tags must have an RCS greater than $-40 \mathrm{~dB}$. Then, the E-shape scatter is a better candidate for authentications purposes, and a $2 \times 2$ arrangement is adopted to increase the RCS level. The final dimensions of the E-structure can be seen in Fig. 4.

Again, the $\mathrm{E}_{2 \times 2}$ structure was duplicated two times in $\mathrm{x}$ - and y-directions in order to increase the RCS level, the simulation comparison among the E-structures is shown in Fig. 5. The obtained RCS of designed $\mathrm{E}_{4 \times 4}$ is shown in Fig. 6. Based on the backscattering pattern, a RCS level higher than $-40 \mathrm{~dB}$ can be obtained for two angles: $\phi_{1}=0^{\circ}$ and $\phi_{2}=90^{\circ}$. Indeed, the structure was developed to favor the backscattering power at these two specific angles. For that, a spacing between the elements greater than $\lambda / 2$ was adopted.

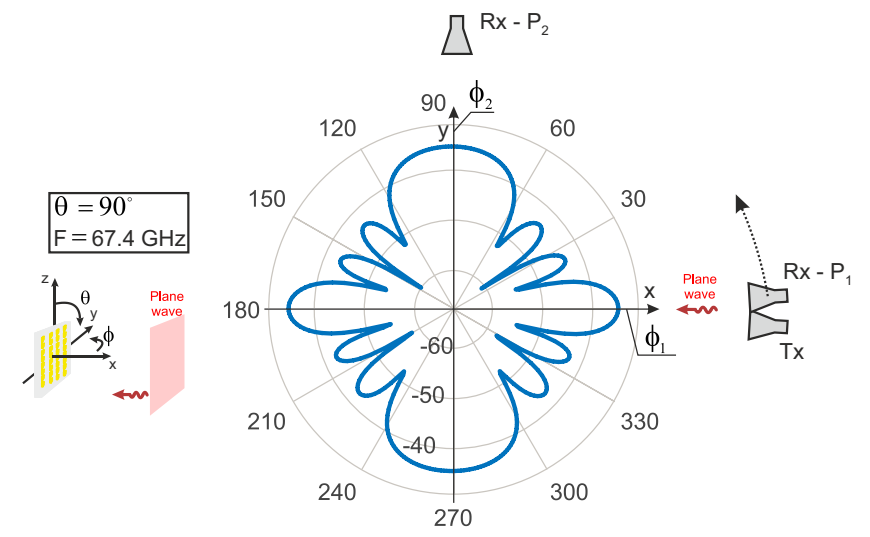

Fig. 6. Simulated RCS polar diagram in $\mathrm{dB}$ of the $E_{4 \times 4}$ tag at $67.4 \mathrm{GHz}$. The measured angles $\phi_{1}=0^{\circ}$ and $\phi_{2}=90^{\circ}$ are highlighted. The incident wave angle is zeros degree; the reception probe is varying from 0 to 360 degree. Both the excitation and reception probes are z-polarized.

\section{EXPERIMENTAL SETUP AND MEASUREMENTS}

A set of 16 tags was fabricated on the same substrate. It is important to note that all tags come from the same digital file, the same substrate, and share the same mask and fabrication process. The measurements were performed with an Agilent N5222A $(0.01 \mathrm{GHz}-26.5 \mathrm{GHz})$ PNA with Virginia Extensions (VDI modules) to operate from $65 \mathrm{GHz}$ to $72 \mathrm{GHz}$. The VDI module is a frequency multiplier combined with a mixer with a $\mathrm{WR}_{15}$ wave-guide output connected to horn antennas on co-polarization configuration. The measurement setup can be seen in Fig. 7. The tag is positioned inside a thin piece of foam. For each measurement, the tag is removed and a new one is placed in the same position to guarantee repeatability. As it is a critical aspect for such approach, the positioning error was studied. A dedicated foam substrate, as shown in Fig. 7, has been specifically fabricated to reduce significantly the positioning error. The tags were placed at a minimum distance of $15 \mathrm{~cm}$ from the antennas and a bi-static configuration is used with time-gating for reducing the multipath interference and decreases the clutter contributions.

The repeatability of the measurement (including positioning error) is the first test. The magnitude of the $S_{21}$ parameters is shown in Fig. 8 where the same $\mathrm{E}_{4 \times 4}$ tag is measured five times. For each measurement, the tag is removed from the foam and placed at the same position. The EM responses for $\phi_{1}=0^{\circ}$ and $\phi_{2}=90^{\circ}$ are shown in Fig. 8(a) and 


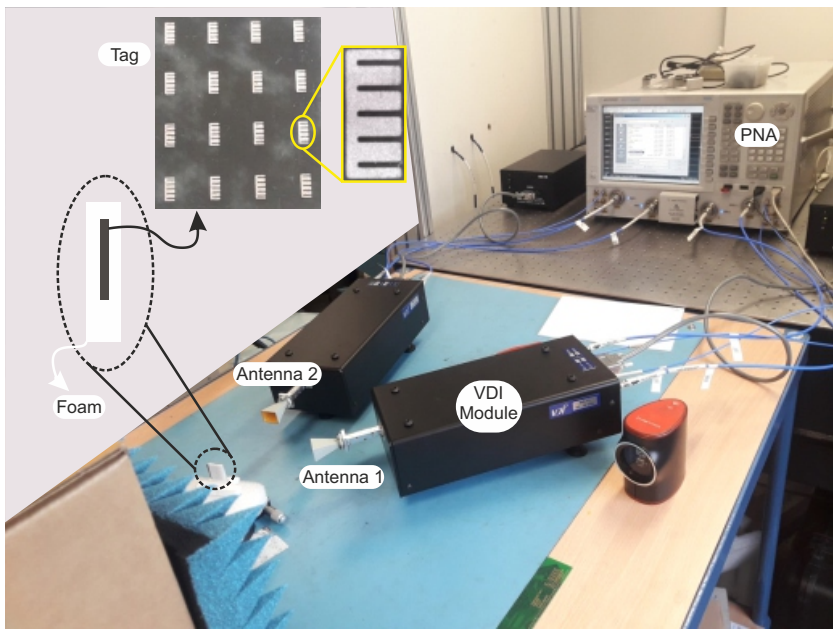

Fig. 7. Setup for V-band measurements in office environment. Bi-static configuration is used, both antennas have co-polarization orientation.

Fig. 8(b), respectively. The general shape of the signal (mainly the amplitude) differs between the two angles. As expected, this means that the contribution of the structure mode is not the same. However, the same resonance can be observed in both cases, which denotes that the backscattered fundamental mode due to the resonant structure is present on all the responses.

The 16 different tags EM response for $\phi_{1}=0^{\circ}$ and $\phi_{2}=90^{\circ}$ are depicted in Fig. 9(a) and Fig. 9(b), respectively. Contrary to Fig. 8 where all the curves were very close to each other, Fig. 9 shows curves that differ from each other. For example, the maximum of each peak apex is no longer at the same frequency. These measurements are the first step of the authentication procedure and it represents the database of Fig. 2(a). The next step is to define a metric to differentiate each response and to evaluate the PE.

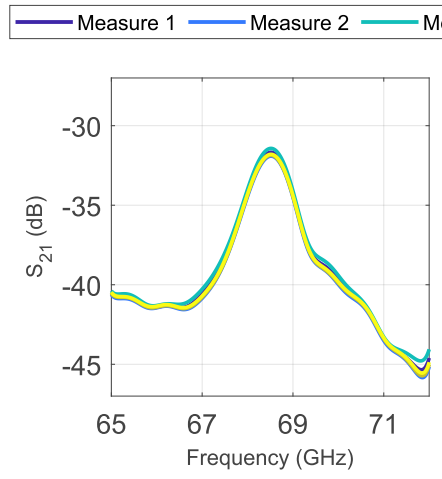

(a)

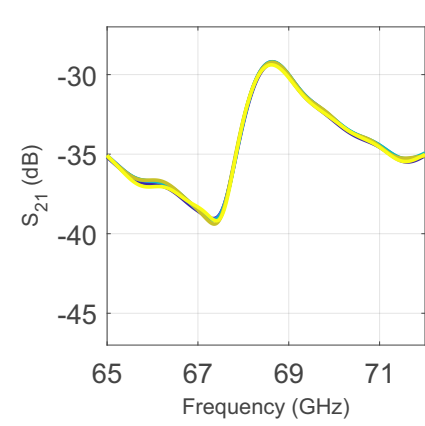

(b)
Fig. 8. Repeatability measurements of one the 16 fabricated tag $\mathrm{E}_{4 \times 4}$, each color corresponds to a $S_{21}$ parameter measured five times at (a) $\phi_{1}=0^{\circ}$ and (b) $\phi_{2}=90^{\circ}$.

\section{EVALUATION OF THE ERROR PROBABILITY OF THE AUTHENTICATION APPROACH}

Machine Learning (ML) has been widely applied to several problems aiming to perform pattern recognition or improve predictions by methods to infer (or learn) decisions rules

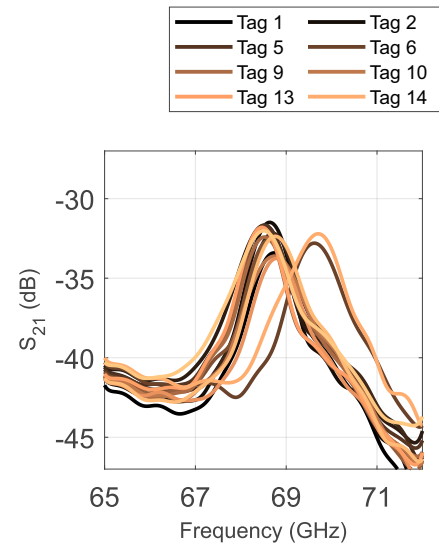

(a)
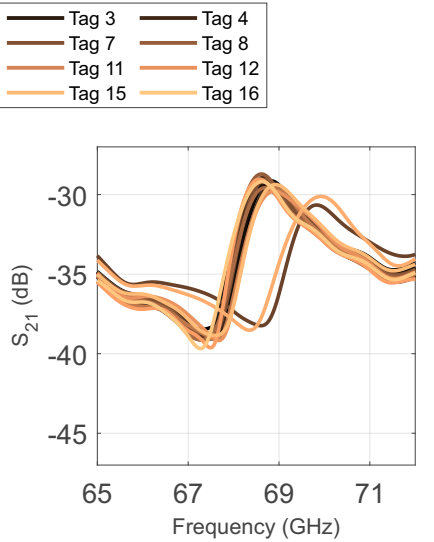

(b)
Fig. 9. The 16 EM tags responses. (a) Tags responses measured at $\phi_{1}=0^{\circ}$ and (b) $\phi_{2}=90^{\circ}$.

from data samples. In particular, ML formulation attempts to answer the following issue: given an observation data-set (tags) of finite objects and the associate results (similarity coefficients), how to build one efficient decision rule able to predict associated results with new entries? In this respect, two classes are constructed from the repeated measurements and the measurements among tags. Then a threshold is estimated to authenticate chipless tags with a minimum PE.

\section{A. ML Approach Classification}

In practice, ML chooses the rule which optimizes a chosen criterion. For instance, this involves determining function parameters [29]. Consecutively, two cases are possible on a linear classification approach: the linearly separable and non-linearly separable data case. The two cases are shown in Fig. 10(a) and Fig. 10(b), respectively. In the separable data case, the technique to construct the optimal separating boundary belonging two classes is equivalent to find the hyperplane described by $\mathbf{H}=\left\{\phi \in R^{2}: \mathbf{w}^{T} \phi=b\right\}$, where $\mathbf{w}$ is a normal vector to the hyperplane, $b$ represents the offset of the separating hyperplane and $\phi=\left(\phi_{1}, \phi_{2}\right)$ concerns the similarity coefficients, which is the input of the problem. The method optimizes the largest margin defining the hyperplane. Graphically, the aforementioned classifier divides the space into two spaces each corresponding different classes, as shown in Fig. 10.

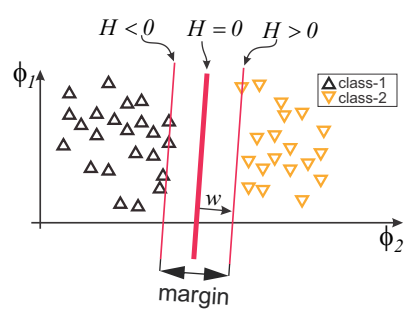

(a)

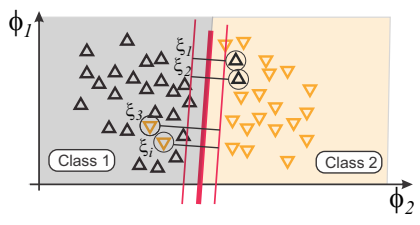

(b)
Fig. 10. Classifier for completely separable case and non-separable case, (a) separable case with decision boundaries from the threshold and (b) nonseparable case with slack variable $(\xi)$. 
Most applications deal with cases where classes are not completely separable by a linear classifier, i.e., misclassifications are envisaged. In this way, a positive slack variable is introduced, which relaxes the constraints seen previously in a completely separable case in order to allow some classification errors, as depicted in Fig. 10(b). The parameter $\xi$ is introduced in the case of linearly separable equation, hence $y_{i}\left(\mathbf{w}^{T} \phi+b\right) \geq 1-\xi_{i}$, where $y_{i} \in\{-1,1\}$ and, $i=0, \ldots, m$ specifies the classification penalty on the data-set. Thus, the algorithm seeks to maximize the margin by optimizing for each constraint a positive error $\xi_{i}$ as small as possible. Once the margin is defined (threshold), new classification entries are possible, as it can be seen in Fig. 10(b). For example, given a couple of measurement $\phi_{1}$ and $\phi_{2}$ of a tag to be authenticated, similarity coefficients are obtained by comparison with the database. If these similarity values are below to the threshold $(\mathbf{H}=0)$, then it belongs to class 1 or the class 2 contrarily.

\section{B. Intra- and Inter-tag Classes definition}

The first step is to compute the similarity coefficients. Consecutively, cosine similarity $\cos \kappa=\operatorname{Re}\left(\mathbf{u} \cdot \mathbf{v}^{*}\right) /(\|\mathrm{u}\| \cdot\|\mathrm{v}\|)$ is computed as a metric to compare the signals, where $\mathbf{u}$ and $\mathbf{v}$ are the two signals to be compared, which are complex data $\left(S_{21}\right.$ parameter previously measured). The operator $(*)$ represents the complex conjugate. Cosine similarity is a measure of similarity between two vectors of complex data. Note that this method is not affected by amplitude signals scaling.

The intra-tag similarity coefficients are computed by the comparison among the repetitive measurements of the same chipless tag, as shown in Fig. 11(a). In the same way, the inter-tag coefficients are obtained by comparing measurements among the tags, as illustrated in Fig. 11(b).
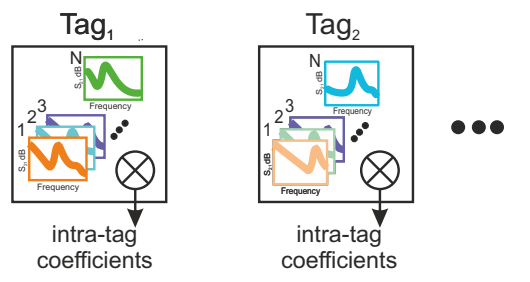

(a)

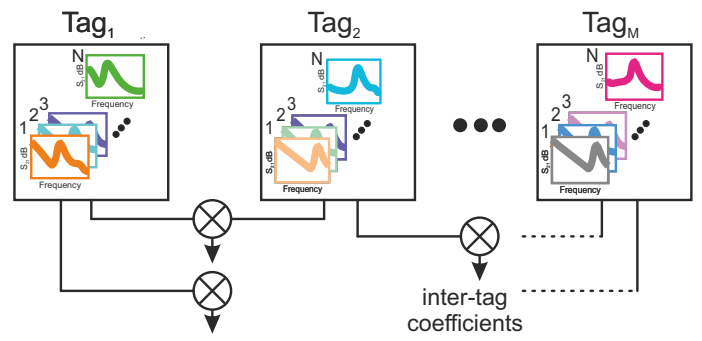

(b)

Fig. 11. Intra-tag and Inter-tag representation with $\mathrm{N}$ measurements and $\mathrm{M}$ tags, (a) intra-tag coefficients $\mathrm{V}=\mathrm{M} . \mathrm{C}_{2}^{\mathrm{N}}$ and (b) $\mathrm{B}=\mathrm{N}^{2} \cdot \mathrm{C}_{2}^{\mathrm{M}}$ inter-tags coefficients are possible for one measured angle.

By taking the similarity coefficients of intra-tag and intertag distributions, these values are mapped on a 2-D plan of coordinate $\left(\mathrm{S}_{\text {intra }}\left(\phi_{1}\right) ; \mathrm{S}_{\text {intra }}\left(\phi_{2}\right)\right)$ or $\left(\mathrm{S}_{\text {inter }}\left(\phi_{1}\right) ; \mathrm{S}_{\text {inter }}\left(\phi_{2}\right)\right)$, where $\mathrm{S}$ is the similarity function for the intra-tag class or inter-tag comparison, considering each angle. This process is depicted in Fig. 12, where each triangle corresponds to a distribution pair for different angles. Thus, once the threshold is defined, new entries can be classified.

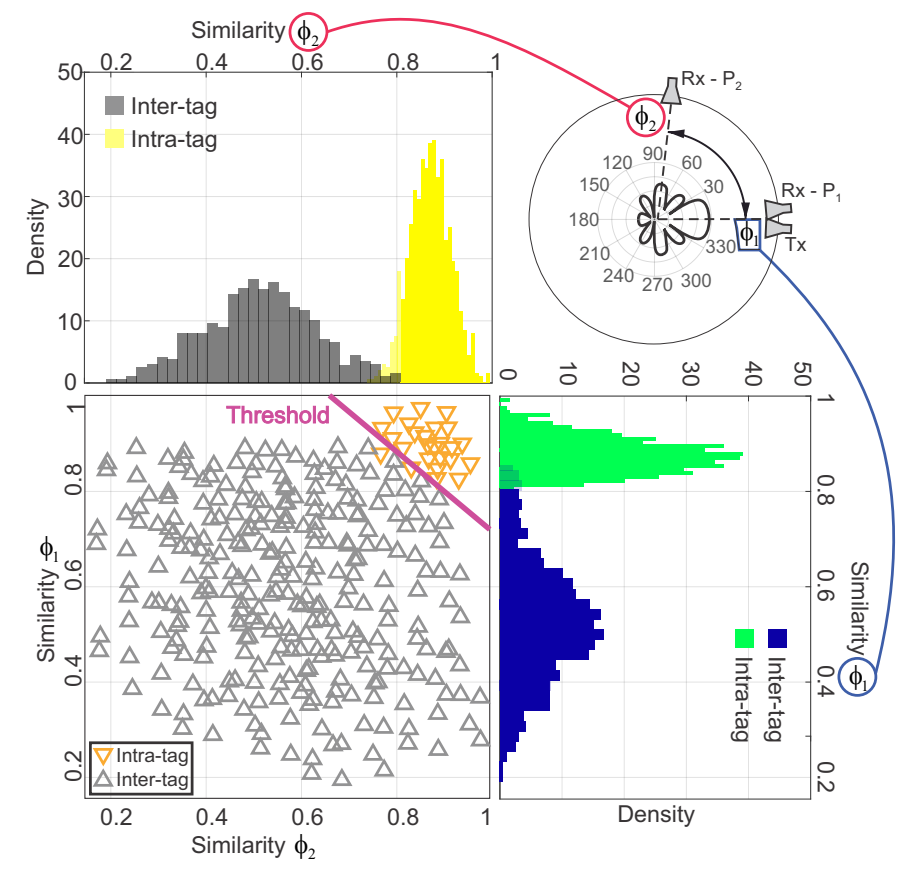

Fig. 12. The intra-tags Mass Distribution Functions (MDF) are computed by the comparison among the repetitive measurements of the same chipless for each angle tag, the coefficients $\left(\mathrm{S}_{\text {intra }}\left(\phi_{1}\right) ; \mathrm{S}_{\text {intra }}\left(\phi_{2}\right)\right)$ and $\left(\mathrm{S}_{\text {inter }}\left(\phi_{1}\right) ; \mathrm{S}_{\text {inter }}\left(\phi_{2}\right)\right)$ are combined on 2-D mapping.

Afterward, new entries are compared with the database for a given angle, as shown in the insets of Fig. 13. Firstly, concerning the tag to authenticate, the measurement is done at $\phi_{1}$, thus compared with whole database fields for the same angle $\phi_{1}$ previously established. The highest similarity coefficient is retained. Secondly, the same procedure is performed considering the angle $\phi_{2}$. Finally, the couple values $\max \left(\phi_{1}, \phi_{2}\right)$ are the entries for the $\mathbf{H}$ threshold function, which geometrically represents the hyperplane. Then, maximum similarity coefficients are computed resulting in 1 if the EM response belongs to the database or -1 otherwise.

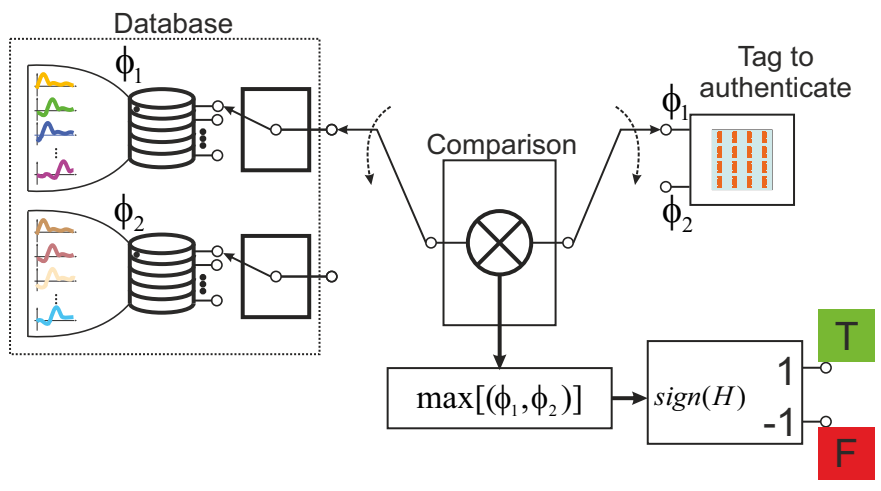

Fig. 13. Comparison among tag measurement for each angle and respective database stored measured angle. 


\section{Performance evaluation}

To authenticate the tags, a threshold using the previously described linear classification approach has been defined. The $\left(\mathrm{S}_{\text {intra }}\left(\phi_{1}\right) ; \mathrm{S}_{\text {intra }}\left(\phi_{2}\right)\right)$ and $\left(\mathrm{S}_{\text {inter }}\left(\phi_{1}\right) ; \mathrm{S}_{\text {inter }}\left(\phi_{2}\right)\right)$ probabilities are shown in Fig. 14. The intra-tag coefficients yield a distribution near to 1 on both axes, which means a good agreement if considering measurements done at the same tag, i.e., a high similarity between the measurements is obtained. In this sense, inter-tag coefficients are dispersed, which indicates a good differentiation between different tag measurements. The misclassification cases are highlighted with the red circle in Fig. 14. It occurs when classes are assigned to a different category than the one it belongs to. The fabricated tags, that share the same substrate, lead to a PE of $1 \%$. Misclassifications are noticed, most of them are false positives (i.e. inter-tag coefficients are in the intra-tag region).

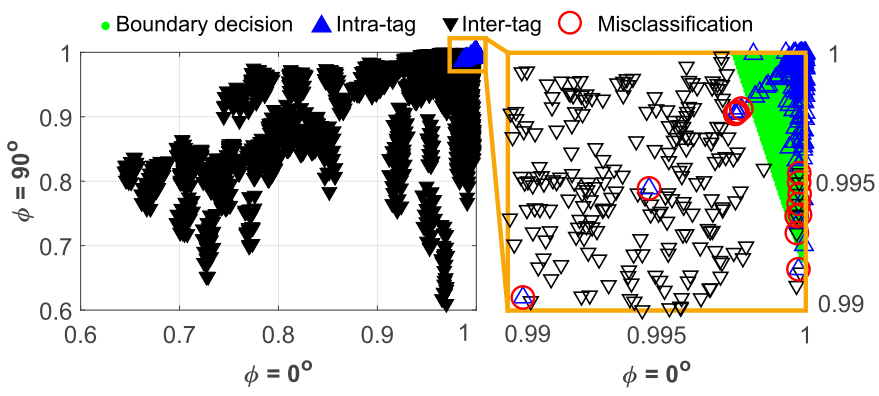

Fig. 14. $\left(\mathrm{S}_{\text {intra }}\left(\phi_{1}\right) ; \mathrm{S}_{\text {intra }}\left(\phi_{2}\right)\right)$ and $\left(\mathrm{S}_{\text {inter }}\left(\phi_{1}\right) ; \mathrm{S}_{\text {inter }}\left(\phi_{2}\right)\right)$ representation and decision boundary performed by linear estimator, some misclassification can be seen.

In this context, the algorithm demonstrates good performance for authentication purposes, besides considering that all the tags have been fabricated at the same time, as reviewed in Table I. As each fabrication process has its own kind of uncertainty, PE can significantly be decreased if tags coming from different fabrications are considered (tags fabricated at a different time even by the same manufacturer). Few studies have been reported using chipless authentication. Moreover, these reported methods have a PE higher than the one reported in this work, as shown in Table I.

TABLE I

CHIPLESS RFID TAG TECHNIQUES FOR AUTHENTICATION

\begin{tabular}{|c|c|c|c|c|}
\hline Ref. & $\begin{array}{c}\text { Different } \\
\text { realizations }\end{array}$ & $\begin{array}{c}\text { Number of } \\
\text { angles }\end{array}$ & $\begin{array}{c}\text { Frequency } \\
(\mathrm{GHz})\end{array}$ & PE \\
\hline$[27]$ & Yes & 1 & $3.1-10.6$ & $3 \%$ \\
\hline$[30]$ & No & 1 & $3.1-10.6$ & $32 \%$ \\
\hline This work & No & 1 & $65-72$ & $5 \%$ \\
\hline This work & No & 2 & $65-72$ & $1 \%$ \\
\hline
\end{tabular}

\section{EVAluATION OF THE TEST BENCH NON-SYSTEMATIC ERROR}

Non-systematic errors are not predictable and cannot be removed through traditional calibration methods; these errors are inherent to every measurement system. Thus, the knowledge of the test bench non-systematic errors is essential for the targeted application. New measurements were performed to evaluate the influence of these non-systematic errors, such as temperature variations, tag positioning and test bench disassembly and reassembly. Furthermore, three new databases for each angle $\left(\phi=0^{\circ} ; \phi=90^{\circ}\right)$ were enrolled, besides the already studied $S_{21}$ repeatability measurements.

\section{A. Temperature stability}

In practical applications, the response of the whole test bench is affected by substantial drift that are mainly related to ambient temperature changes. To evaluate the influence of ambient temperature variations on the tag's response, the same tag has been measured at different temperatures. The digital temperature sensor (TES-1365) was used to monitor the ambient temperature variations in the environment. Initially, the system is turned on and all the measurements are done in an interval of 15 hours. $S_{21}$ measurements are taken every two minutes at both angles $\left(\phi=0^{\circ} ; \phi=90^{\circ}\right)$. The measured temperature profiles under the chosen angles $\left(\phi=0^{\circ} ; \phi=90^{\circ}\right)$ can be seen in Fig. 15. The two profiles are different because the measurements are carried out in an uncontrolled temperature environment and are not done the same day. As depicted in Fig. 15, the Vector Network Analyzer (VNA) is turned on at $\left(t_{0}=0 \mathrm{~s}\right)$ and warms during the first 40 minutes. Then, the room temperature varies in a range of $1^{\circ} \mathrm{C}$ around $26^{\circ} \mathrm{C}$.

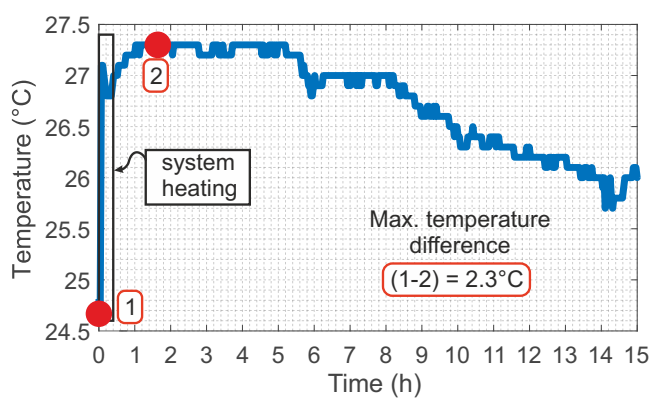

(a)

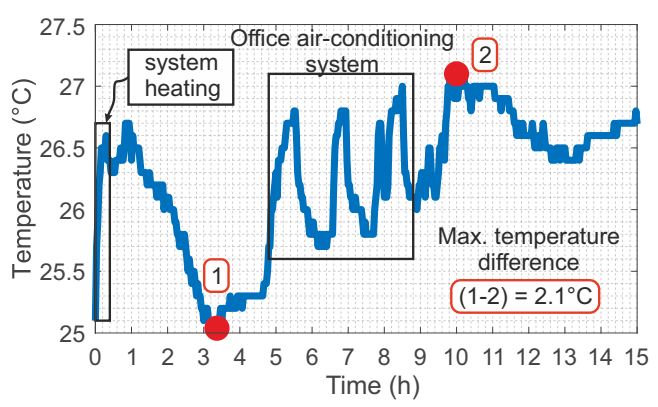

(b)

Fig. 15. The temperature profiles of the measurements the two different angles: (a) $\phi=0^{\circ}$, (b) $\phi=90^{\circ}$. The two measurements were done at different times.

The peak apex amplitude variations (corresponding to the tag resonance extracted from the S21 parameter) versus the time for both angles are shown in Fig. 16. An amplitude variation of $0.1 \mathrm{~dB}$ for $\phi=0^{\circ}$ and $0.06 \mathrm{~dB}$ for $\phi=90^{\circ}$ is observed. To evaluate the effect of these variations on the error probability, the cosine similarity has been computed between the complex responses corresponding to points (1) and (2) 
shown on each figure. A similarity of 0.9986 and 0.9991 is obtained from the highlighted points in Fig. 16(a) and Fig. 16(b), respectively. These similarity coefficients do not induce to a false negative reading. So, the additional amplitude drift will not induce an error.

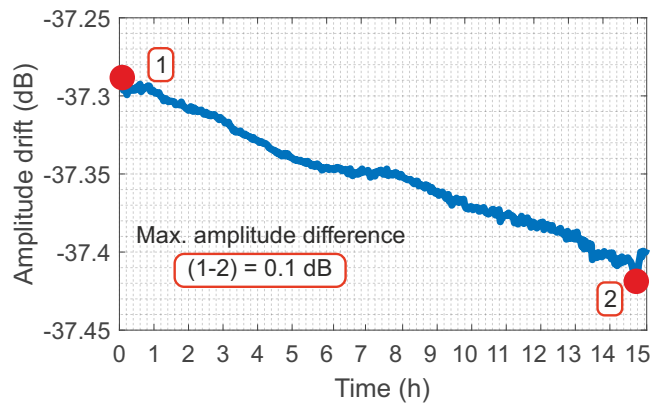

(a)

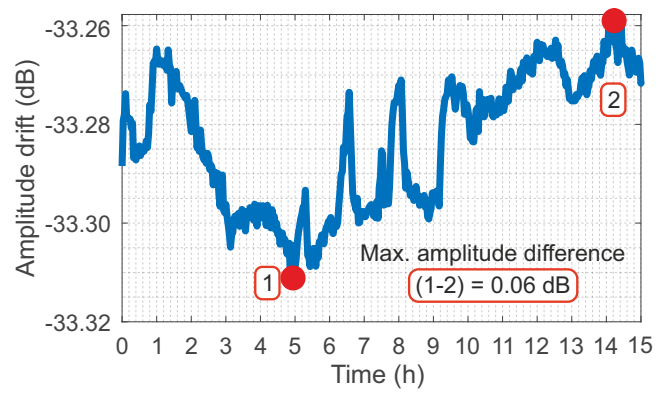

(b)

Fig. 16. Amplitude peak variation as function of time for (a) $\phi=0^{\circ}$ and (b) $\phi=90^{\circ}$. The temperature profiles of the measurements are given in Fig. 15 .

The frequency drift of the peak apex (resonance frequency) versus the time for both angles are shown on Fig. 17. A drift of $10 \mathrm{MHz}$ for $\phi=0^{\circ}$ and $31 \mathrm{MHz}$ for $\phi=90^{\circ}$ is observed. To evaluate the effect of this variation on the error probability, the cosine similarity comparison between the complex responses corresponding to points (1) and (2) shown on Fig. 17 has been evaluated. A similarity of 0.9988 and 0.9982 is obtained from the highlighted points in Fig. 17(a) and Fig. 17(b), respectively. This similarity is higher than the threshold (0.9827) defined in the article considering a single angle. So, the frequency drift will not induce an error.

The drift errors are inherent to the millimeter-wave test bench. However, the impacts are insignificant considering a stable temperature environment (which is the nominal configuration of use of such a measuring device). Thus, the system must be implemented in a well-known and controllable/constant temperature environment.

\section{B. Tag positioning sensibility}

Another relevant factor to analyze the non-systematic errors is the tag/support positioning errors. Therefore, the sensitivity inherent in each measurement angle by support rotation/translation movement is evaluated. As depicted in Fig. 18, the support is placed on a platform that can perform rotations and translation movements with high accuracy.

$S_{21}$ measurements are performed at the two considered angles $\phi=0^{\circ}$ and $\phi=90^{\circ}$. The initial position $P_{0}$ is saved

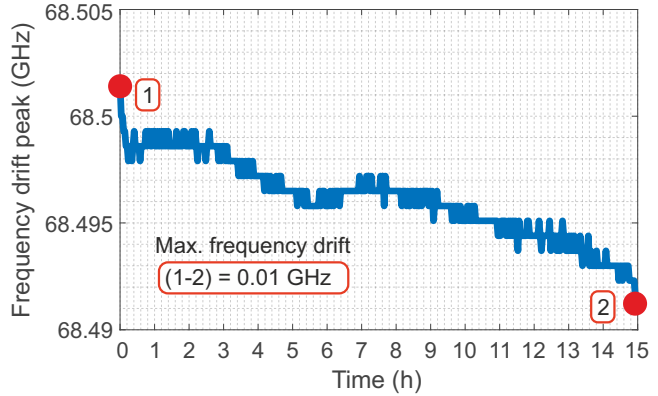

(a)

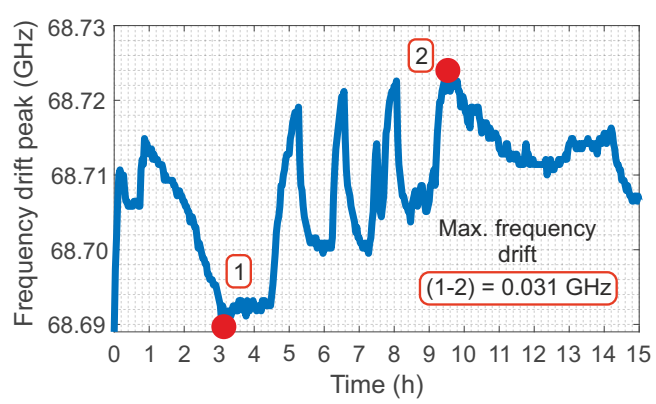

(b)

Fig. 17. Frequency peak variation as function of time for (a) $\phi=0^{\circ}$ and (b) $\phi=90^{\circ}$. The temperature profiles of the measurements are given in Fig. 15.

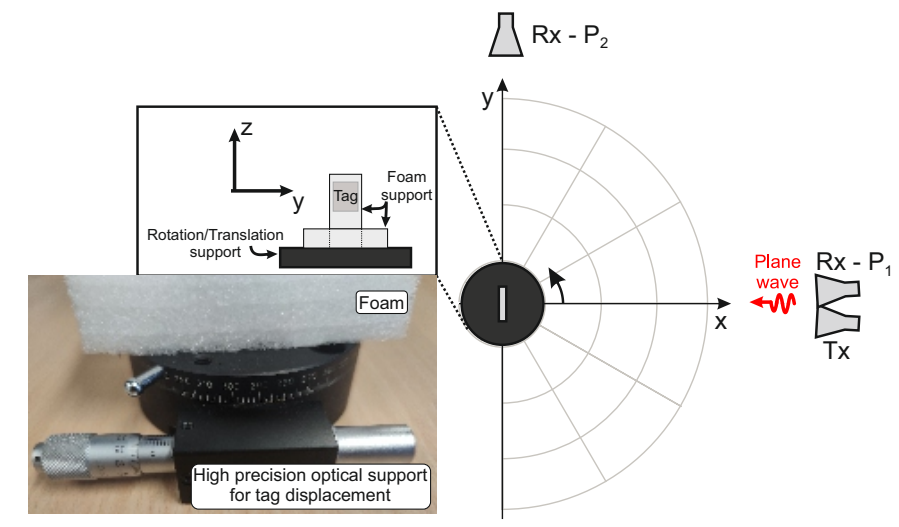

Fig. 18. Support for tag rotation/translation. The rotation is around the support axis itself, whereas the translation is by changing the positions following the y-axis.

for comparison purposes. Then, the similarity coefficients due to the tag translation for a sequence of different positions, relatively to the initial position, is computed. As depicted in Fig. 19, the measurement at the angle $\phi=90^{\circ}$ is more sensitive to small variations imposed on the tag support for both rotation and translation displacements. In this way, the test bench is significantly affected by variations in case of measurements at $\phi=90^{\circ}$, both in rotation and translation, as shown in Fig. 19(a) and Fig. 19(b), respectively. Then, concerning the translation movement, which is equivalent to the issue of tag positioning inside the foam support, the tag can be shifted until $0.1 \mathrm{~mm}$ without negatively affecting the similarity coefficients. However, rotation movements have to be controlled using non-rotated support. If this condition cannot be ensured, the measurements will not be able to be 
repeated in case of our targeted application.

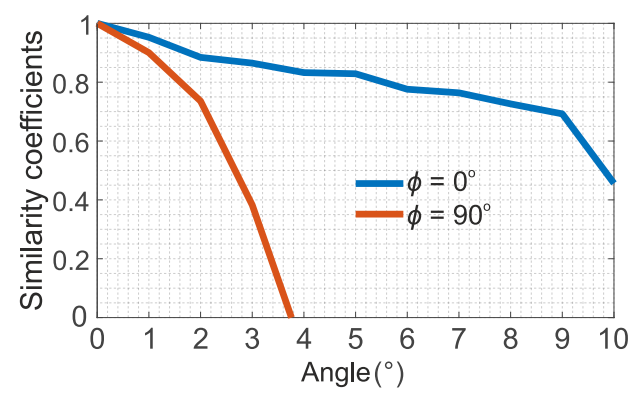

(a)

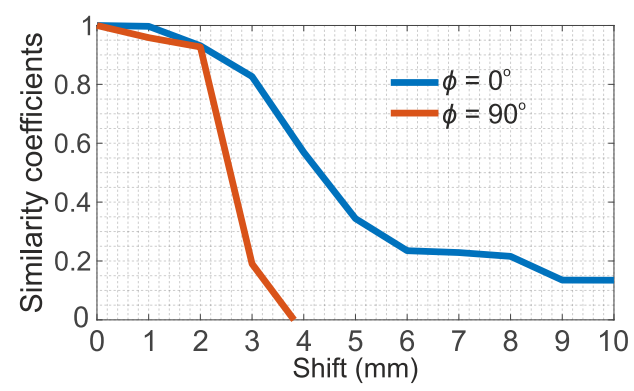

(b)

Fig. 19. Similarity coefficients considering the (a) rotation of tag support and (b) the translation of tag support.

\section{New database enrollment}

Finally, to assess the reproducibility of the measurement test bench, three new databases were enrolled. For each database enrollment, the test bench was completely disassembled and reassembled. Before each database enrollment, a measurement without the tag is performed. This empty measurement makes it possible to consider some of the variations that may exist from one test bench to another.

Fig. 20 shows the measurement results of the same tag (tag measurement minus empty measurement of the corresponding test bench) for the three test benches realized. A maximum amplitude difference among the measurements is approximately $0.7 \mathrm{~dB}$ for $\phi=0^{\circ}$, as can be seen in Fig. 20(a) and, a maximum variation of $0.8 \mathrm{~dB}$ is noted considering $\phi=90^{\circ}$, as depicted in Fig. 20(b). Most of these variations are related to the fact that despite the efforts to reproduce the measurement benches identically, small disparities (cable positions, cable tightening, etc.) have an impact on the Sparameters. However, this impact remains low (lower than $0.8 \mathrm{~dB}$ on the tag measurement). The frequency shift seen in Fig. 20 represents a negligible change $\left(10^{-5}\right)$ on the similarity coefficients. Then, it indicates that when the databases are compared individually the measures are quite similar.

After databases enrollment, the PE for each database is evaluated. Table II shows the whole PE associated with each database. Therefore, considering the databases enrollment at $\phi=0^{\circ}$, the non-systematic errors have a low impact on the measurements which leads to close results between the databases. This is different with the measurements performed

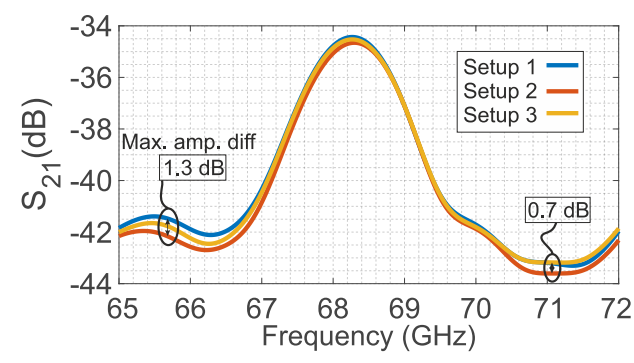

(a)

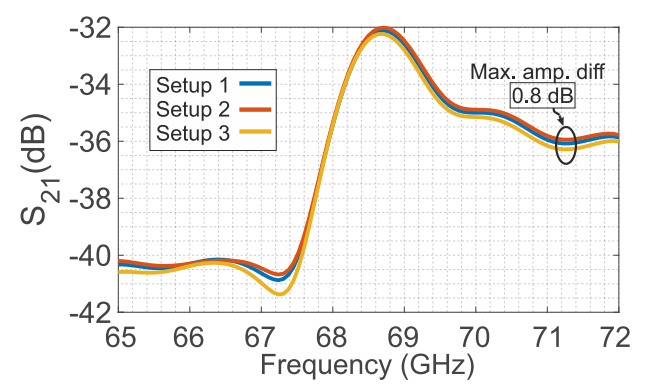

(b)

Fig. 20. The tag measurement considering the test bench that has been disassembled and reassembled three times (noted setups here): (a) $\phi=0^{\circ}$ and (b) $\phi=90^{\circ}$.

at $\phi=90^{\circ}$, which are highly impacted by position misalignments. Thus, the test bench could be disassembly and after reassembly without constraints at $\phi=0^{\circ}$. Nonetheless, when measurements at $\phi=90^{\circ}$ are performed, the system should not be disassembled to keep acceptable performance.

TABLE II

PROBABILITIES OF ERROR ASSOCIATED TO EACH DATABASE.

\begin{tabular}{|c|c|c|c|}
\hline Angles & DE $(\%)$ & Database 1 & Database 3 \\
\hline$\phi=0^{\circ}$ & 2.36 & 2.69 & 2.75 \\
\hline$\phi=90^{\circ}$ & 5.90 & 8.24 & 8.18 \\
\hline$\phi=0^{\circ} ; \phi=90^{\circ}$ & 0.6 & 0.9 & 0.5 \\
\hline
\end{tabular}

\section{Comparison among databases}

Beyond the PE comparisons considering the same database, the comparison between the different databases is done. It allows to evaluate the PE deviation when the tag measurements were carried out with a measurement bench that has been assembled/disassembled. Only measurements retrieved at $\phi=0^{\circ}$ are evaluated, since at $\phi=90^{\circ}$ it has been seen that measurements repeatability is not accurate enough.

Again, the cosine similarity has been used as a metric for comparison between the measurements. New classes are defined to the database (DB) comparison: i) the intra-DB class, that concerns the comparison between the same tag measures when different databases are analyzed, ii) the interDB class that relates the different tags measures comparison considering two different databases. Then, the PE associated to database comparison is evaluated. This analysis allows knowing if the fact of assembling and disassembling the test bench changes the probability of error. Then, the changes in 
the probability of error compared to only one database means that the supposed increase of probability of error is due to the non-systematic errors present in the assembly/disassembly of the set. The Table III shows the PE associated to each database comparison.

TABLE III

PE COMPARISON BETWEEN DATABASES.

\begin{tabular}{|c|c|c|}
\multicolumn{1}{l|}{} & Database 2 & Database 3 \\
\hline Database 1 & $8.33 \%$ & $8.88 \%$ \\
\hline Database 2 & & $6.21 \%$ \\
\hline
\end{tabular}

The observable increase of the PE indicates that nonsystematic errors due to assembly/disassembly are present. However, these non-systematic errors may be reduced by using the measurement of a reference object. Indeed, the same object (like a simple metal plate) can be measured one time with each setup and used as a reference target to remove part of assembly error between different database. With this measurement and the empty measurement, it would be possible to calibrate all the tag measurements in order to compute a RCS value [18] which is a quantity independent of the measurement bench. For all these reasons, the test bench assembly/disassembly can be performed at $\phi=0^{\circ}$.

Given that the method proposed in this article that combines two different angles, the whole PEs are in the range of $0.6 \%-1 \%$. Additionally, considering measurements repeatability issue, the system must fulfill the following conditions:

- Measurements must be performed in a well-known and controllable temperature environment, which should not vary more than $2^{\circ} \mathrm{C}$;

- The support must be able to provide an accuracy of at least $0.1 \mathrm{~mm}$ in translation and must not rotate (rotation angle less than $1^{\circ}$ )

- The system can be disassembled only if measurements at $\phi=0^{\circ}$ are considered.

\section{CONCLUSIONS}

A method using millimeter-wave tags for authentication applications purpose was evaluated. The tags were designed, manufactured and analyzed. The backscattered E-field of these tags is highly sensitive to physical parameter variations naturally imposed by the manufacturing process. The approach of duplicating the number of resonators to form a tag was used to overcome the RCS low level. A set of 16 chipless tags were developed in the V-band, these tags consist of backscattering a unique EM response exploiting the inherent fabrication random side. The backscattered RCS level is satisfactory for real environment reading measurements even without specific calibration. Different angles were measured to obtain the tag authenticity; the exploitation of the backscattered pattern is a promising method for authentication applications. Furthermore, the associated drift error was analyzed to each angle. Therefore, considering the databases enrollment at $\phi=0^{\circ}$, the non-systematic errors have a low impact on the measurements, contrary to the measurements performed at $\phi=90^{\circ}$, since the measurements are highly impacted by position misalignments. Therefore, the test bench assembly/disassembly can be performed at $\phi=0^{\circ}$ only. However, the following conditions must be fulfilled: tag measurements in an environment where the temperature is stabilized, the tag support must be able to provide an accuracy of at least $0.1 \mathrm{~mm}$ in translation. Statistical analysis was discussed, the intra-tag and inter-tag distribution coefficients from each angle are mapped on a 2D plan looking hyperplane estimation for binary classification and a PE around 1\% was estimated, the PE improvement is remarkable when compared to the measurement using a single angle (5\%). Other tags can be developed to maximize backscattering in more than two different directions. In such a case, multiple measurements can continue to reduce the PE significantly. However, the complexity of obtaining good thresholds will increase as well. Future work intends to implement a lower cost solution, using bio-based and recyclable techniques from the paper industry.

\section{ACKNOWLEDGEMENT}

The authors would like to acknowledge the University Grenoble Alpes for financially supporting of this project AUSTRALE via the ANR program.

\section{REFERENCES}

[1] N. Ratha and R. Bolle, Automatic Fingerprint Recognition Systems. Springer Science \& Business Media, Oct. 2003.

[2] W. Stallings, Cryptography and network security: principles and practice, seventh edition ed. Boston: Pearson, 2014.

[3] P. Scanlon, I. O. Kennedy, and Y. Liu, "Feature extraction approaches to RF fingerprinting for device identification in femtocells," Bell Labs Technical Journal, vol. 15, no. 3, pp. 141-151, Dec. 2010.

[4] R. M. Gerdes et al., "Device Identification via Analog Signal Fingerprinting: A Matched Filter Approach." The Internet Society, 2006.

[5] A. C. Polak, S. Dolatshahi, and D. L. Goeckel, "Identifying Wireless Users via Transmitter Imperfections," IEEE Journal on Selected Areas in Communications, vol. 29, no. 7, pp. 1469-1479, Aug. 2011.

[6] S. Dolatshahi, A. Polak, and D. L. Goeckel, "Identification of wireless users via power amplifier imperfections," in 2010 Conference Record of the Forty Fourth Asilomar Conference on Signals, Systems and Computers, Nov. 2010, pp. 1553-1557.

[7] K. Merchant et al., "Deep Learning for RF Device Fingerprinting in Cognitive Communication Networks," IEEE Journal of Selected Topics in Signal Processing, vol. 12, no. 1, pp. 160-167, Feb. 2018.

[8] V. Lakafosis et al., "RF Fingerprinting Physical Objects for Anticounterfeiting Applications," IEEE Transactions on Microwave Theory and Techniques, vol. 59, no. 2, pp. 504-514, Feb. 2011.

[9] L. Bolotnyy and G. Robins, "Physically Unclonable Function-Based Security and Privacy in RFID Systems," in Fifth Annual IEEE International Conference on Pervasive Computing and Communications (PerCom'07), Mar. 2007, pp. 211-220.

[10] S. Devadas et al., "Design and Implementation of PUF-Based "Unclonable" RFID ICs for Anti-Counterfeiting and Security Applications," in 2008 IEEE International Conference on RFID, Apr. 2008, pp. 58-64.

[11] S. Devadas, "Non-networked RFID-PUF authentication," US Patent US8 683 210B2, Mar., 2014. [Online]. Available: https://patents.google. $\mathrm{com} /$ patent/US8683210B2/en?oq=US8683210B2

[12] E. Perret, Radio Frequency Identification and Sensors: From RFID to Chipless RFID. John Wiley \& Sons, Dec. 2014.

[13] R. S. Nair and E. Perret, "Folded Multilayer C-Sections With Large Group Delay Swing for Passive Chipless RFID Applications," IEEE Transactions on Microwave Theory and Techniques, vol. 64, no. 12, pp. 4298-4311, Dec. 2016.

[14] A. Chamarti and K. Varahramyan, "Transmission Delay Line Based ID Generation Circuit for RFID Applications," IEEE Microwave and Wireless Components Letters, vol. 16, no. 11, pp. 588-590, Nov. 2006.

[15] R. de A. Junior, G. Fontgalland, and R. A. Rodrigues, "Low cost folded chipless tag for millimeter-wave applications," in 2018 IEEE International Symposium on Antennas and Propagation USNC/URSI National Radio Science Meeting, Jul. 2018, pp. 1499-1500. 
[16] I. Jalaly and I. Robertson, "Capacitively-tuned split microstrip resonators for RFID barcodes," in 2005 European Microwave Conference, vol. 2, Oct. 2005, pp. 4 pp. -1164 .

[17] S. Preradovic et al., "Multiresonator-Based Chipless RFID System for Low-Cost Item Tracking," IEEE Transactions on Microwave Theory and Techniques, vol. 57, no. 5, pp. 1411-1419, May 2009.

[18] A. Vena, E. Perret, and S. Tedjini, "Chipless RFID Tag Using Hybrid Coding Technique," IEEE Transactions on Microwave Theory and Techniques, vol. 59, no. 12, pp. 3356-3364, Dec. 2011.

[19] A. Vena, E. Perret, and S. Tedjni, "A Depolarizing Chipless RFID Tag for Robust Detection and Its FCC Compliant UWB Reading System," IEEE Transactions on Microwave Theory and Techniques, vol. 61, no. 8, pp. 2982-2994, Aug. 2013.

[20] O. Rance et al., "Toward RCS Magnitude Level Coding for Chipless RFID," IEEE Transactions on Microwave Theory and Techniques, vol. 64, no. 7, pp. 2315-2325, Jul. 2016.

[21] A. Vena, E. Perret, and S. Tedjini, "Design of Compact and AutoCompensated Single-Layer Chipless RFID Tag," IEEE Transactions on Microwave Theory and Techniques, vol. 60, no. 9, pp. 2913-2924, Sep. 2012.

[22] N. Barbot, O. Rance, and E. Perret, "Angle Sensor Based on Chipless RFID Tag," IEEE Antennas and Wireless Propagation Letters, vol. 19, no. 2, pp. 233-237, Feb. 2020.

[23] E. Perret, "Displacement Sensor Based on Radar Cross-Polarization Measurements," IEEE Transactions on Microwave Theory and Techniques, vol. 65, no. 3, pp. 955-966, Mar. 2017.

[24] K. Yang, D. Forte, and M. M. Tehranipoor, "UCR: An unclonable chipless RFID tag," in 2016 IEEE International Symposium on Hardware Oriented Security and Trust (HOST), May 2016, pp. 7-12.

[25] Z. Ali et al., "Potential of chipless authentication based on randomness inherent in fabrication process for RF and THz," in 2017 11th European Conference on Antennas and Propagation (EUCAP), Mar. 2017, pp. 2559-2563.

[26] _ - "Chipless RFID Tag Discrimination and the Performance of Resemblance Metrics to be used for it," in 2018 IEEE/MTT-S International Microwave Symposium - IMS, Jun. 2018, pp. 363-366.

[27] — , "Detection of Natural Randomness by Chipless RFID Approach and Its Application to Authentication," IEEE Transactions on Microwave Theory and Techniques, vol. 67, no. 9, pp. 3867-3881, Sep. 2019.

[28] — , "Authentication Using Metallic Inkjet-Printed Chipless RFID Tags," IEEE Transactions on Antennas and Propagation, vol. 68, no. 5, pp. 4137-4142, May 2020.

[29] T. Hastie, R. Tibshirani, and J. Friedman, The Elements of Statistical Learning: Data Mining, Inference, and Prediction, Second Edition. Springer Science \& Business Media, Aug. 2009.

[30] Z. Ali et al., "Potential of chipless authentication based on randomness inherent in fabrication process for RF and THz," Ph.D. dissertation, Paris, France, Mar. 2017. [Online]. Available: https://hal.archives-ouvertes.fr/hal-01800579

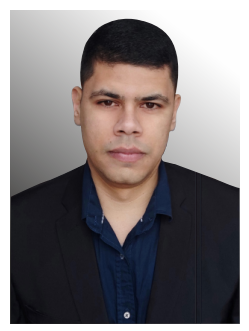

Raymundo de Amorim Junior received the B.S. degree in electrical engineering and M.S. degree in telecommunications and electronics both from Universidade Federal de Campina Grande, Campina Grande, Brazil, in 2016 and 2018, respectively. From 2013 to 2018, he was a member Applied Electromagnetics and Microwave Laboratory (LEMA). $\mathrm{He}$ is currently pursuing the Ph.D. degree with the Laboratoire de Conception et d'Intégration des Systèmes, Univ. Grenoble Alpes, Valence, France. His research interests include antenna characterisation, antenna array characterisation, reverberant chambers, RF circuits and chipless RFID millimeter- and submillimeter-wave applications.

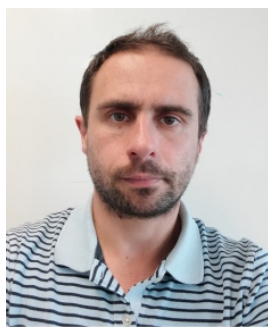

Romain Siragusa Romain Siragusa was born in Paris, France. He received the Engineering degree and the Master's degree from the Institute National Polytechnique de Grenoble (Grenoble-INP), Grenoble, France, in 2006, and the Ph.D. degree from the same institute at the Laboratoire de Conception et d'Intégration desSystèmes (LCIS), Valence, France, all in electronic engineering. From 2009 to 2010, he joined the "Commissariat À L'énergie Atomique" (CEA) as a Post DoctoralFellow, where he studied the high impedance surface for integrated antenna on silicon. Since 2011, he has been an Associate Professor with Grenoble-INP Esisar/LCIS. His research interests include leaky-wave structures and chipless radio-frequency identification (RFID).

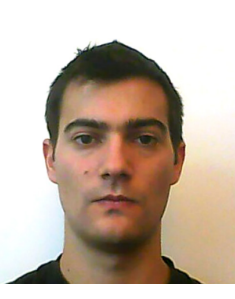

Nicolas Barbot Nicolas Barbot (Member, IEEE) received the M.Sc and Ph.D. degrees in 2010 and 2013 from the Université de Limoges, Limoges, France. His Ph.D. work was focused on error-correcting codes for the optical wireless channel at the Xlim Laboratory, Limoges, France. He also realized a post-doctoral work in joint source-channel decoding at the LSS Laboratory, Gif-sur-Yvette, France. Since September 2014, he has been an Assistant Professor with the Grenoble Institute of Technology, Université Grenoble Alpes, Valence, France. His research interests at the LCIS, Université Grenoble Alpes, include backscattering communications, RFID, and chipless RFID. More recently, he investigates the use of chipless tag as low-cost, batteryless, and robust sensors.

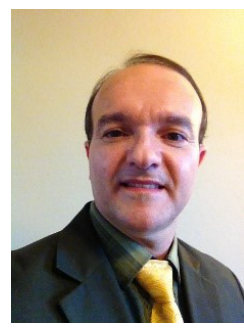

Prof. Dr. Glauco Fontgalland (S'97-A'99-M'00-SM'07) was born in Brazil. Graduated (1990) and M.S. degree (1993) in Electrical Engineering from the Universidade Federal de Campina Grande (UFCG), Brazil. He has a $\mathrm{PhD}$ in Electronics from Toulouse Institut National Polytechnique - ENSEEIHT (1999),

Toulouse, France, where his Thesis work was nominated for the Leopold Escande Award 1999. From 2010 to 2012 he was visiting scholar at ElectroScience Laboratory at the Ohio State University - OSU, USA. Currently, He is a Full Professor at the UFCG, Campina Grande, BR, where he develops research on: electromagnetic modeling, EMC, EMI, ESD, RFID, UWB, propagation, and antennas for various applications. He has published more than 200 papers in journal and conferences. He is past IEEE AP-S chapter chair Northeast Session, R9, and chair of the IEEE AP-S 2021 student design contest (SDC) and member of IEEE AP-S 2020/2021 Field Awards Evaluation. Since 2019, he is an Associate Editor at IEEE Latin America Transactions. Prof. Dr. Fontgalland is a member of the Sociedade Brasileira de Micro-ondas e Optoeletrônica (SBMO), Sociedade Brasileira de Eletromagnetismo (SBMag), Sociedade Brasileira de Microeletrôncia (SBMicro), and The applied Computacional Eletromagnetics Society (ACES). 


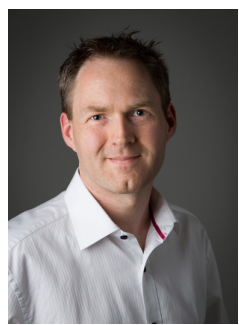

Etienne Perret (S'02-M'06-SM'13) received the Eng. Dipl. degree in electrical engineering from the Ecole Nationale Supérieure d'Electronique, d'Electrotechnique, d'Informatique, d'Hydraulique, et des Télécommunications, Toulouse, France, 2002, and the M.Sc. and Ph.D. degrees in electrical engineering from the Toulouse Institute of Technology, Toulouse, in 2002 and 2005, respectively. From 2005 to 2006 , he held a post-doctoral position with the Institute of Fundamental Electronics, Orsay, France. In 2006, he was appointed Associate Professor of electrical engineering at Grenoble INP - Institute of Engineering Univ. Grenoble Alpes, France. From 2014 to 2019, he has been a Junior Member with the Institut Universitaire de France, Paris, France, an institution that distinguishes professors for their research excellence, as evidenced by their international recognition. From 2015 to 2020, he has been an Appointed Member of the French National Council of Universities. He has authored or co-authored more than 200 technical conferences, letters and journal papers, and books and book chapters. He holds several patents. His works have generated more than 3000 citations. His current research interests include electromagnetic modeling of passive devices for millimeter and submillimeterwave applications, and wireless communications, especially RFID and chipless RFID, and also include advanced computer-aided design techniques based on the development of an automated codesign synthesis computational approach. Dr. Perret is a Technical Program Committee member of the IEEE International Conference on RFID, the IEEE RFID TA; and a member of the IMS Technical Paper Review Committee. He was a recipient of several awards like the MIT Technology Review's French Innovator's under 35 in 2013, the French Innovative Techniques for the Environment Award in 2013, the SEE/IEEE Leon Brillouin Award for his outstanding achievement in the identification of an object in an unknown environment using a chipless label or tag in 2016, the IEEE MTT-S 2019 Outstanding Young Engineer Award and the Prix Espoir IMT - Académie des sciences in 2020. He was a Keynote Speaker and the Chairman of several international symposiums. Etienne Perret was awarded an ERC Consolidator Grant in 2017 for his project ScattererID. 